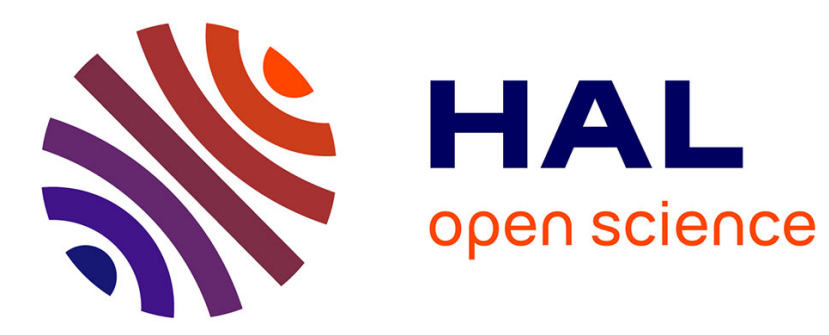

\title{
Violences conjugales, violences théoriques. La psychanalyse à l'épreuve du genre
}

\author{
Thamy Ayouch
}

\section{To cite this version:}

Thamy Ayouch. Violences conjugales, violences théoriques. La psychanalyse à l'épreuve du genre. Cliniques méditerranéennes, 2013, 88 (2013), pp.19-34. halshs-01003864

\section{HAL Id: halshs-01003864 https://shs.hal.science/halshs-01003864}

Submitted on 10 Jun 2014

HAL is a multi-disciplinary open access archive for the deposit and dissemination of scientific research documents, whether they are published or not. The documents may come from teaching and research institutions in France or abroad, or from public or private research centers.
L'archive ouverte pluridisciplinaire HAL, est destinée au dépôt et à la diffusion de documents scientifiques de niveau recherche, publiés ou non, émanant des établissements d'enseignement et de recherche français ou étrangers, des laboratoires publics ou privés. 


\section{Violences conjugales, violences théoriques. La psychanalyse à}

\section{l'épreuve du genre}

Thamy Ayouch, Leila Salomão de la Plata Cury Tardivo

Thamy Ayouch

Psychanalyste, Maître de Conférences en Psychopathologie Clinique, Université de Charles de Gaulle - Lille 3, Professor Visitante do Exterior, Universidade de São Paulo.

Rua Doutor Brasilio Machado, 103, Apto 23, 01230-010, São Paulo, SP, Brésil.

thamy.ayouch@gmail.com

Leila Salomão de la Plata Cury Tardivo

Professeur de Psychologie Clinique, Universidade de São Paulo.

Universidade de São Paulo, Instituto de Psicologia, Av. Prof. Mello Morãoes 1721, Cidade Universitária - 05508-030 São Paulo, SP, Brésil

tardivo@usp.br 
«I am asham'd that women are so simple

To offer war where they should kneel for peace;

Or seek for rule, supremacy, and sway,

When they are bound to serve, love, and obey ${ }^{1}$.

Ces propos, que Shakespeare, dans La Mégère apprivoisée, met ironiquement dans la bouche d'une Katharina assujettie, pourraient être invoqués par bien des auteurs de violences conjugales, soucieux de naturaliser la position de soumission dont sortirait la femme qu'ils battent. C'est parfois cette même naturalisation de «la femme » et du "féminin », moyennant quelques modifications de rôle assigné, que l'on se surprend à lire dans certaines approches théoriques de ces violences conjugales.

Plus de quatre siècles après l'écriture de La Mégère apprivoisée, cette conception est loin d'avoir disparu, et l'extension historique et géographique des violences conjugales reste tristement considérable. La majeure partie de ces violences touche les femmes, que les contextes socio-culturels les plus variés assignent souvent au rôle de «servir, aimer et obéir ». Si les postures théoriques diverses, de la sociologie à la psychanalyse, prennent acte de cette scandaleuse situation, certaines interprétations ne manquent pas de refléter bien des assignations de genre.

Mon propos consistera ici à analyser certaines théorisations psychanalytiques, à la lumière d'une expérience clinique acquise auprès de femmes souffrant de violences

\footnotetext{
${ }^{1}$ W. Shakespeare, The Taming of the Shrew, Acte 5 , scène 5.
} 
conjugales, dans la banlieue de São Paulo. Je mène, conjointement avec Leila Tardivo, Professeure à l'Université de São Paulo, une recherche clinique auprès de la Casa Sofia, institution d'accueil psychologique de femmes victimes de violences conjugales. J'y accompagne, depuis plus d'un an, plusieurs patientes dans un dispositif de psychothérapie analytique individuelle, et d'autres dans le cadre d'une psychothérapie de groupe.

Cette recherche vise à définir certaines modalités de correspondance entre la psyché et le champ social. La psyché reprendrait, dans ses formes d'édification, l'organisation socio-politique. A leur tour, les formes des cette organisation de la collectivité seraient le reflet de la structure de la psyché. L'inconscient est modelé par le social et le politique, le politique infiltré de désir inconscient.

Cette dimension sociétale d'inscription de la psyché et l'historicité de sa structuration, n'exemptent pas alors la théorisation qui tente de rendre compte de la psyché. Comment, en effet, aborder certains phénomènes définis historiquement, culturellement, socialement, par une théorisation aux prétentions d'universalisme éternitaire, oublieuse de son inscription historique, sociale, culturelle, imaginaire?

Poser la question de l'inscription historique d'un regard, d'une approche clinique et d'une théorisation est, à mon sens, une démarche spécifiquement psychanalytique. Quel abord psychanalytique clinique peut-on alors faire de la violence conjugale, en considérant l'inscription social-historique de la psyché, et celle de la théorisation? Quelle approche clinique et théorique peut être développée pour éviter que la théorie 
n'exerce, à son tour, une violence symbolique, en reproduisant un paradigme de violence de genre non-analysé ?

Pointant une «crise de la psychanalyse » dans les États d'âme de la psychanalyse. Adresse aux États Généraux de la Psychanalyse, Jacques Derrida posait la question de savoir ce que la révolution psychanalytique met en crise, mais aussi ce qui la met en crise. Deux forces étaient ici décelées : à la résistance à la psychanalyse, très tôt explicitée par Freud, s'ajoute la « résistance auto-immunitaire de la psychanalyse à son dehors comme à ellemême ». L'omission de l'historicité de sa théorisation et de son inscriptions dans des formations discursives, l'abord prescriptif de la sexuation et la de sexualité, sont, à mon sens, autant de formes de cette résistance. Ainsi donc, pourrait-on demander, quels remaniements cliniques et théoriques l'appréhension des violences conjugales impliquet-elle, pour contrer cette « résistance auto-immunitaire » de la psychanalyse ?

\section{La spécificité d'une approche analytique}

Considérer l'inscription historique d'un regard, d'une approche et d'une théorisation est une démarche spécifiquement psychanalytique. Dans une nietzschéenne interrogation de la libido sciendi, la pensée psychanalytique met un point d'honneur à indiquer les errances possibles de toute théorie menacée par la folie de son dogmatisme. L'originalité de l'expérience analytique de la cure est supposée ouvrir la voie à un rapport tout autre à la connaissance, dans une articulation où, plus que l'énoncé, c'est l'énonciation qui importe. Interroger sur la posture depuis laquelle est émis un discours, sur son adresse, et sur son inscription historique est ainsi une démarche 
psychanalytique. Mais c'est une interrogation dont la pratique et, surtout, la théorie psychanalytique ne peuvent s'exempter, et qu'elle doivent retourner contre ellesmêmes.

En quoi consisterait alors une approche psychanalytique des violences conjugales?

Il y a de ce sujet divers abords psychologiques - systémiques, gestaltiques, phénoménologiques, - qui consistent souvent à décrire les phénomènes, parfois dans une visée de scientificité, à y déceler des régularités. Ainsi parle-t-on des divers types de violence - physique, psychologique, morale, patrimoniale, sexuelle, - ou identifie-t-on, dans une approche confinée à l'observation phénoménologique, un cycle répétitif de violence, dans l'alternance d'une phase de tension, d'une phase d'explosion et d'une phase de lune de miel. Ce cycle se répète souvent jusqu'à culminer dans une blessure grave ou la mort de la victime.

Le propre d'une approche analytique ici est de viser, par-delà l'observabilité de ces phénomènes, la logique des processus inconscients qui les caractérisent, et les structures psychiques et relationnelles qu'ils convoquent. Ces mécanismes inconscients procèdent de destins pulsionnels intrapsychiques et articulent des processus de subjectivation heureuse ou malheureuse. Ils ne se limitent toutefois pas à des identités intrapsychiques de victime et d'agresseur ontologisées, mais convoquent une irréductible dimension relationnelle. Ils visent la dynamique par laquelle les mécanismes de défenses d'un couple se radicalisent pathologiquement, ou deviennent obsolètes, culminant parfois dans des modes relationnels pervers narcissiques. 
En 1923, Freud définit la psychanalyse dans une triplicité de visées voulues indissociables : discours de l'inconscient, clinique et théorie. Le terme désigne ainsi un «procédé d'investigation des processus psychiques, qui autrement sont à peine accessibles » - l'inconscient, - une « méthode de traitement » basée sur cette dernière, et «une série de conceptions psychologiques acquises par ce moyen $»^{2}$, la métapsychologie. Cette définition reste fondamentale, faisant de la psychanalyse non point d'abord une théorie mais une méthode liée à un objet, l'inconscient, rendu positif par la procédure de recherche et la technique psychanalytique.

De cette méthode résulte un dispositif clinique et une technique: l'écoute psychanalytique, association libre et attention flottante, vise, par la règle d'abstinence, à toujours respecter la liberté du sujet (ni décider pour lui, ni lui infliger un dressage). Ce sujet est divisé, il est défini par une excentricité à lui-même à lui-même, livré, pour reprendre des termes lacaniens, au signifiant et à la structure. Cela signifie que tout refoulement présuppose un sujet et son refus, désirant, d'une certaine association signifiante rejetée. A cela tient la division du sujet : celui qui refuse est le même que celui qui souffre des conséquences de son refus, et c'est bien du sujet lui-même que l'approche analytique attend qu'il prenne la mesure de ce qu'il a lui-même refusé. C'est ici ce qui probablement différencie éthiquement, cliniquement et théoriquement un ensemble de procédures qui proposent de délivrer le sujet d'un mal qui lui est étranger, et la psychanalyse, pour qui le sujet est impliqué dans la souffrance dont il se plaint.

2 S. Freud, « Psychanalyse et "théorie de la libido" », in Résultats, idées, problèmes II (1921-1938), trad. sous la direction de J. Laplanche, Paris, P.U.F., 1985, p 51. 
Il convient donc, lors du suivi de patients souffrant de violence conjugale, de se demander qui parle et à qui cette parole est adressée, en examinant la posture subjective articulée dans la fois la plainte en séance et dans la position dans le couple violent. Il s'agit également d'appréhender la dynamique relationnelle violente et la fantasmatisation complémentaire qu'elle convoque, et d'examiner ce que vient signifier le maintien de la violence ou son retour. La perspective analytique consiste à interroger l'implication irréductible du sujet dans ce qui lui arrive, et à révéler ainsi la jouissance que la répétition met en œuvre. Toutefois, cette interrogation n'a pas pour visée de résonner en incrimination, et encore moins en assignation ou en prescription de posture subjective.

C'est néanmoins, semble-t-il, le travers que connaissent certaines approches psychanalytiques de la violence conjugale.

\section{Violence des théorisations}

Littéralisant ou imaginarisant des textes freudiens ou lacaniens, bien des interprétations psychanalytiques se font fort d'expliquer la violence subie par une femme par son irréductible féminité, où l'envie du pénis le dispute à un masochisme féminin essentialisé, sous le règne d'un CEdipe dont la résolution a-morale (car ultérieure au complexe de castration) se paie d'un dangereux penchant au sacrifice.

Dans sa description du complexe d'CEdipe féminin ${ }^{3}$, Freud présente comme inversée chez les femmes la relation entre OEdipe et castration. Si, pour les garçons, le complexe

\footnotetext{
3 Dans «La disparition du complexe d'CEdipe» (1923), «Quelques conséquences psychiques de la différence anatomique des sexes » (1925) ou « La sexualité féminine » (1931)
} 
d'CEdipe disparaîtrait sous l'effet du complexe de castration, ce dernier, chez les filles, le précèderait et l'annoncerait. Celles-ci connaîtraient d'abord un «Penis-Neid», et changeraient ensuite d'objet, troquant la mère qui les a condamnées à la féminité pour un père susceptible de remplacer le pénis manquant par une promesse d'enfant.

L'imaginarisation de cette théorisation reste liée, il convient de l'indiquer, à des représentations de genre et d'identités propres à l'inscription sociale, historique et culturelle de ces textes. Freud le rappelle, en accentuant la dimension métaphorique de ces descriptions et des outils métapsychologiques qu'il en déduit, et en affirmant que tous les individus possèdent des traits masculins et féminins à la fois, du fait de la bissexualité constitutive de la psyché. Toutefois, et malgré la différence notable entre la Vienne du début du vingtième siècle et le contexte social, culturel, et anthropologique actuel, d'habiles théoriciens ne se privent guère d'entendre littéralement ces descriptions et d'en faire de dogmatiques assignations de destins psychiques.

Si le masochisme, ajoutent d'aucuns, est lié à un sentiment de culpabilité inconsciente procédant de la masturbation infantile (comme le soutient Freud dans «On bat un enfant»), la «complaisante » inscription d'une femme dans une relation violente proviendrait du fantasme d'être battue-passivée-fécondée par le père.

Ces expertes articulations de concepts, bien trop prises dans le narcissisme de leur construction pour s'ouvrir à toute singularité clinique, semblent caricaturales. Elles n'en sont pas moins communes, chez certains théoriciens improvisés de la violence conjugale. 
Citons ici, à titre d'exemple paradigmatique, l'article « Bate-se numa mulher - questões sobre a violência e o feminino » ${ }^{4}$, redigé par Maria Carolina Bellico Fonseca, Maria Isabel Sá et Vanessa Campos Santoro.

Rappelant certains développements freudiens sur la sexualité féminine, l'article statue que la castration permet la reconnaissance de la différence entre les sexes et l'instauration d'un ordre sexuel, où le phallus règne en maître. C'est, après Lacan, au tour de Freud d'être littéralisé, lorsque les auteures désignent trois voies de développement de l'être-femme, dans cette sortie de la castration : la première conduirait à l'inhibition sexuelle et à la névrose, la seconde à une modification du caractère dans le sens du complexe de masculinité, et la troisième, seule, à une « féminité normale ».

Confrontée à la castration, poursuit l'article, la petite fille peut se désintéresser du plaisir sexuel et se révéler ainsi impuissante devant la vie, par un refoulement trop violent de l'envie du pénis. Et d'illustrer cela par la situation d'une première patiente. Celle-ci, que son mari oblige à dormir sur un fauteuil dans le salon, en trempant son côté du lit lorsqu'il est en colère, se voit gratifiée du commentaire suivant, qui laisse perplexe sur l'usage fait des termes lacaniens :

« Aveuglée par les attributs phalliques qu'elle prête au mari, M.E. se détourne du manque qui la constitue et ne peut glisser vers la recherches d'objets de substitution $»^{5}$

Se pose ici la question de la pertinence de l'accueil de cette patiente par un discours de refus de la castration - du reste, parfaitement littéralisée et imaginarisée, comme le

\footnotetext{
${ }^{4}$ Maria Carolina Bellico Fonseca, Maria Isabel Sá et Vanessa Campos Santoro, « Bate-se numa mulher questões sobre a violência e o feminino », Reverso. Revista de psicanálise, $\mathrm{n}^{\circ} 48$, Septembre 2001.

${ }^{5}$ Fonseca, M. C., Isabel, S. M., \& Vanessa, C. S. (2001). Bate-se uma mulher- questões sobre a violência e o feminino. Reverso. Revista de psicanalise , 48. Nous traduisons.
} 
montre la suite de l'article. Gageons, à lire les auteures, que les femmes connaissent des violence conjugale pour ne pas se conformer à l'unique voie saine de résolution de la castration conduisant à assumer une posture féminine. C'est bien justement, alors, qu'elles mériteraient cette violence, pour ne pas être assez femmes - éternel féminin, qu'on ne s'étonnera pas, au demeurant, de ne voir renvoyé à aucune relativité sociohistorique.

La seconde voie, sortie par le complexe de masculinité où la femme «vivra le fantasme d'être un homme » et « rivalisera avec les hommes », est illustrée par la situation d'une autre patiente qui explique en ces termes, se passant de commentaires, pourquoi elle bat son mari :

« Je travaille, paie les factures et il boit toute la journée. Peut-être qu'en le battant, je lui apprendrai ce que c'est qu'être un homme ${ }^{6}$.

La troisième voie, où, harmonieusement, l'envie du pénis cède la place à un objet qui s'y substitue, conduit bien heureusement à une féminité où se fait la représentation du manque, et à la recherche d'un équivalent du phallus dans la chaine symbolique. Elle permet que la femme «trouve le meilleur substitut (du phallus) lorsqu'elle est renvoyée à l'autre sexe », mais en visant dans cette rencontre sexuelle le tout naturel but de la reproduction. Une troisième patiente illustre ainsi bien malheureusement ce destin raté : qu'elle soit battue par son mari alcoolique tient à ceci qu'elle ne peut pas avoir d'enfant, du fait d'un avortement effectué pendant son adolescence.

${ }^{6}$ Ibid. 
Que la théorie prétendument analytique s'avise de prescrire la bonne voie de la sexuation et l'heureux destin de la sexualité tendue vers la seule reproduction, et dénonce, mieux que le Vatican, la pratique de l'avortement, ne sert pas de la meilleure façon la clinique.

Plus encore, il est, poursuit l'article, une «provocation féminine » qui trouverait son expression dans le désir toujours insatisfait de la femme, et la convoque à un «plus-dejouir », renvoyant à la jouissance de l'Autre, qui, pour être interdite de l'inconscient, déclenche comme seule réponse l'hors-la-loi de la pulsion destructive. C'est ce que montre un patient parlant de sa femme en ces termes :

«Elle ne me fout pas la paix. Il ne lui manque rien, mais elle réclame toujours. C'est qu'elle veut s'en prendre une ! Mais qu'est-ce qu'elle veut, cette femme? ».

Et les auteures d'en déduire que ne sachant plus quoi donner à sa femme, cet homme n'a d'autre recours que de lui donner des coups. Concluons de ces analyses éclairées qu'une femme mérite d'être battue pour être phallique, et ne pas vouloir se laisser castrer.

La féminité est alors présentée comme cet «indicible », qui, d'une certaine manière, expliquerait ici la violence conjugale : portant sur le corps les insignes de la castration, les femmes les rappellent continuellement aux hommes, qui ne savent répondre à ce que veut la femme que par des coups. Somme toute, la castration réelle de la femme, imaginarisée comme défaut de pénis, confronterait les hommes à leur castration propre, à leur manque, et à un plus-de-jouir impossible qui déclencherait irréductiblement leur violence. 
Et voilà pourquoi votre fille est muette - et, partant, votre femme battue...

L'article conclut alors magistralement que c'est le féminin, en tout vivant, qui a besoin d'être battu, dans une équivalence fantasmatique entre être battue par le père et avoir avec lui une relation sexuelle passive.

Bien d'autres exemples pourraient malheureusement être cités, nous nous cantonnerons toutefois à ce morceau choisi. Par-delà l'infatuation de cette lecture, et l'impéritie avérée de ces formules toutes formelles, apparaît ici le point aveugle, l'axe narcissique de toute opération de théorisation, mais aussi sa participation de formations discursives ininterrogées, provoquant ce que Michel Foucault nommait « dispositifs de sexualité » .

Certaines approches proclamées psychanalytiques procèdent ainsi à une essentialisation de la différence sexuelle qui ne provient que d'une assignation de genre, insouciantes de l'ordre sexiste promu par cette naturalisation qui provoque et légitime la violence contre les femmes. Par delà le cynisme clinique ici mis en œuvre, apparaît le problème d'une dogmatisation théorique éloignée de la spécificité des situations et de leur contexte historique.

Ces savantes théories invitent donc à tenter de relire certaines références analytiques, sans les littéraliser8.

\footnotetext{
${ }^{7}$ Foucault, M. (1976). Histoire de la sexualité. La volonté de Savoir. Paris: Gallimard.

${ }^{8}$ Nous pensons ici, entre autres, aux textes freudiens «Un enfant est battu », in Freud, S. (1973). Névrose, psychose, perversion. Paris: P.U.F., et «Le problème économique du masochisme », in Freud, S. (1973). Névrose, psychose, perversion. Paris: P.U.F.
} 


\section{Théorie et violence de genre}

Plus fondamentalement, c'est la question de la différence des sexes et son traitement par la psychanalyse qui est ici convoquée bruyamment dans la clinique et la théorisation de la violence conjugale. Inscrivant la sexualité contre la reproduction en la défonctionnalisant, Freud ne manque pas de provoquer par là une dé-naturalisation de la sexuation aussi. En effet, lorsque la visée de la pratique sexuelle n'est pas le destin biologique de la reproduction, la complémentarité des deux sexes de l'espèce apparaît toute relative.

Plus encore que la relativité d'une complémentarité des sexes, c'est essentiellement leur définition qui semble problématique, les notions de « masculin » et de «féminin » étant, souligne Freud, de part en part « confuses $»^{9}$. La psychanalyse «ne peut (...) élucider» l'essence de ces notions, « elle reprend à son compte les concepts et les met à la base des ses travaux $»^{10}$. Tous les individus humains, « par suite de leur constitution bisexuelle et de leur hérédité croisée, possèdent à la fois des traits masculins et des traits féminins, si bien que le contenu des constructions théoriques de la masculinité pure et de la féminité pure reste incertain ${ }^{11}$.

Freud soutient toutefois, dans un autre texte que «la différence morphologique [doit] se manifester dans des différences dans le développement psychique » ${ }^{12}$. Et de conclure, parodiant Napoléon : «l'anatomie est un destin ${ }^{13}$. La tendance semble radicalisée en 1937, lorsque, dans « Analyse avec fin, analyse sans fin », il souligne que la source la plus puissante de la résistance, en analyse, reste, pour les hommes, la révolte contre l'attitude

\footnotetext{
${ }^{9}$ Freud, S. (1987). Trois essais sur le théorie sexuelle. Paris: Gallimard, Folio Essais.

10 Freud, S. (1973). Psychogénèse d'un cas d'homosexualité féminine. Dans S. Freud, Névrose, psychose, perversion. Paris: P.U.F., p 270.

${ }^{11}$ Ibid., p 132.

${ }_{12}^{12}$ Freud, S. (1969). La disparition du complexe d'Oedipe. Dans S. Freud, La Vie sexuelle. Paris: P.U.F., p 121.

${ }^{13}$ Ibid.
} 
passive ou féminine à l'endroit d'un autre homme, et pour les femmes l'envie du pénis, aspiration à la possession d'un organe génital masculin ${ }^{14}$. La biologie et son déterminisme, auparavant écartés, semblent ici de nouveau convoqués, comme « roc d'origine $»^{15}$.

Le texte freudien est donc pluri-stratifié, comme le montrent les lectures riches en nuances de Monique Schneider ${ }^{16}$. N'en favoriser qu'une interprétation univoque l'appauvrit alors considérablement et provoque la rigidification d'une différence sexuelle essentialisée, naturalisée, et rendue responsable de vicissitudes psychiques universelles.

Plutôt qu'une différence des sexes, la clinique de la violence conjugale n'invite-t-elle pas à envisager ici une intersubjective, et partant, toute sociale différence des genres, qui loin d'être anatomique, est assignée? Comme le souligne Jean Laplanche dans Castration, symbolisations, préalable à la différence des sexes se trouve une différence des genres, du masculin et du féminin, admise sans être théorisée ${ }^{17}$. Cette différence, souvent impensée par la psychanalyse, est, comme le développe Jean Laplanche dans un texte ultérieur, assignée par le social ; c'est, plutôt que l'« identification primitive au père de la préhistoire personnelle», une «identification primitive par le socius de la préhistoire personnelle ${ }^{18}$.

\footnotetext{
${ }^{14}$ Freud, S. (1985). L'analyse avec fin et l'analyse sans fin. Dans S. Freud, Résultats, idées, problèmes. II. Paris: P.U.F., p 267.

${ }^{15}$ Ibid., p 268.

${ }^{16}$ Schneider, M. (2000). Généalogie du masculin. Paris: Aubier et Schneider, M. (2004). Le paradigme féminin. Paris: Aubier.

${ }^{17}$ Laplanche, J. (1980). Problématiques II. Castration, symbolisation. Paris: P.U.F.

${ }^{18}$ Laplanche, J. (2003). Sexual. La sexualité élargie au sens freudien. Paris: P.U.F, p 167.
} 
La clinique de la violence conjugale amène donc à considérer de quelle manière ces assignations de genre sont effectuées dans la société, et plus particulièrement intériorisées par les patient.e.s, mais aussi comment elles peuvent être reproduites par la théorie.

Les théories du genre semblent alors ouvrir un champ prometteur pour la pensée psychanalytique, dans la reconnaissance d'une historicité de ses catégories théoriques, notamment de la différence sexuelle. La perspective de Judith Butler, par exemple, remodèle en profondeur les rapports du sexe et du genre et permet d'appréhender la manière dont peut opérer une violence de genre au sein même de la production théorique tentant de penser les violences conjugales.

Dans Troubles dans le genre, l'opposition entre sexe et genre n'est plus pensée sur le modèle du divorce entre nature et culture. Pas moins que le genre, le sexe est lui aussi une construction sociale: l'anatomie n'est pas un destin mais une fabrication historique ${ }^{19}$. Judith Butler vise ainsi à défaire toute essentialisation de la différence des sexes : il n'y a pas de nature ontologique, de différence des sexes anatomique essentielle qui ne soit toujours et déjà prise dans un sens institué, culturellement et historiquement défini, du genre. Le genre est l'ensemble des moyens discursifs et culturels par lesquelles une "'nature sexuée' ou un 'sexe naturel' est produit et établi dans, un domaine 'prédiscursif' qui précède la culture, telle une surface politiquement neutre sur laquelle intervient la culture après coup ${ }^{20}$. En résulte la notion de performativité de

\footnotetext{
${ }^{19}$ Thomas Laqueur le montre déjà dans Laqueur, T. (1992). La Fabrique du sexe. Essai sur le corps et le genre en Occident. Paris: Gallimard.

${ }^{20}$ Butler, J. (2005). Troubles dans le genre. Le féminisme et la subversion de l'identité. Paris: La découverte, $\mathrm{p}$ 69.
} 
genre, répétition de normes créant, par l'imitation, l'idée d'un modèle original qui n'existe guère hors de cette réitération.

Certains concepts analytiques, littéralisés, ou abordés hors de leur dimension socialhistorique, perpétuent, semble-t-il, cette performativité du genre, la prescrivent, même, en assignant un parcours fléché du développement psychique - castration, OEdipe, assomption de la différence des sexes - qui sert alors a contrario de mode d'explication de l'adversité ou la disgrâce que rencontre le sujet. S'il ou elle souffre, c'est, à en croire certains, un châtiment mérité pour avoir échappé à cette performativité de genre que lui prescrit l'itinéraire d’un enfant castré.

Je soutiendrais, au contraire, que certaines situations de violence conjugale viennent de postures subjectives tentant de se décoller de ces assignations de genre, de défaire la captation imaginaire qu'elles introduisent au sein d'un couple. Y apposer une lecture inscrivant la théorie dans ces assignations de genre revient alors à scotomiser la clinique, et révèle un point aveugle, non analysé, de l'analyste qui, confronté à ces angoissantes contestations du genre, se rabat sur sa théorie sexuelle infantile.

Le concept de phallus, précisément théorisé par Lacan pour lever toute dimension biologisante de la différence des sexes, semble se prêter souvent à ce type de glissement dans une imaginarisation de la théorie, et une prescription de la posture subjective. 


\section{Phallus et semblant}

Dans le texte «La signification du phallus», Lacan définit le phallus eu égard au complexe de castration, mais aussi à «l'installation dans le sujet d'une position inconsciente sans laquelle il ne saurait s'identifier au type idéal de son sexe ni même répondre sans de graves aléas aux besoins de son partenaire dans la relation sexuelle, voire accueillir avec justesse ceux de l'enfant qui s'y procrée $»^{21}$. L'identification du sujet au type idéal de son sexe reste imaginaire, de même que la réponse aux besoins du partenaire dans la relation sexuelle - qui, notons-le, contient étonnamment en germe l'enfant qui s'y procrée. Retenons toutefois que cette visée idéale d'assomption du sexe est paradoxale, antinomique, elle n'advient que par une menace ou une privation.

Le phallus est alors défini par sa fonction : il n'est ni fantasme, ni objet (partiel ou interne), ni organe, pénis ou clitoris, qu'il symbolise toutefois. Le phallus est un signifiant, et non des moindres, «c'est le signifiant destiné à désigner dans leur ensemble les effets de signifié, en tant que le signifiant les conditionne par sa présence de signifiant $»^{22}$.

Les relations entre les sexes sont soumise à la fonction du phallus dans l'être ou l'avoir, dans un rapport imaginaire au phallus irréalisant la relation à l'autre. Le phallus induit ainsi un paraître pour chacun des sexes, une « comédie » dans la recherche par chacun des «manifestations idéales ou typiques des comportements de chacun des sexes $»^{23}$, jusque dans l'acte sexuel. C'est ici le semblant. De ce semblant résultent deux postures de sexuation. La femme entend être désirée pour ce qu'elle n'est pas, et pour paraître

\footnotetext{
${ }^{21}$ Lacan, J. (1958). « La Signification du phallus », in Ecrits. Paris, Seuil, p 685.

22 Ibid., p 693.

23 Ibid.
} 
être le phallus, rejette «une part essentielle de la féminité, nommément tous ses attributs dans la mascarade $»^{24}$. Pour elle, en l'autre convergent demande et désir : le défaut de désir sexuel, la frigidité, serait alors bien mieux toléré par elle que par l'homme.

L'homme, lui, trouvant la satisfaction de sa demande d'amour dans la relation à une femme, ne cèdera pas sur son désir du phallus. Celui-ci « fera surgir son signifiant dans sa divergence rémanente vers 'une autre femme' qui peut signifier ce phallus à divers titres, soit comme vierge, soit comme prostituée ». L'impuissance est alors chez lui bien moins bien supportée.

La question qui ne manque pas de se poser dans la lecture de ce texte est de savoir que sont ces entités, fixées au préalable, de «femme » et d' « homme ». A quoi renvoient-elles dans leur généralité et ne frisent-elle pas l'essentialisation? N'entend-on pas ici, à trop littéraliser la théorie, une série de postures historiques et historicisées rendant l'impuissance plus problématique que la frigidité, la femme moins génitale que l'homme?

Si donc le phallus vient battre en brèche une vision différentialiste de la sexuation, fondée sur une différence des sexes naturalisée, il est toutefois introduit dans un contexte culturel et historique très marqué : la féminité mascarade, l'impuissance masculine moins bien supportée que la frigidité, ou l'homosexualité féminine comme résultat d'une déception sentiraient leur contexte masculiniste et hétérocentré de 1958, si l'on n'accentuait ici, avant tout, sa dimension cruciale de semblant.

\footnotetext{
${ }^{24}$ Ibid., p 694.
} 
Littéralisé, imaginarisé, ce texte pourrait donc donner lieu à une véritable prescription de performativité de genre. Dans un couple - sous-entendu hétérosexuel, - la posture de la femme consistera à incarner le phallus sans l'avoir, et se voit prescrire d'être garante du désir de l'autre, qui n'est pas sans l'avoir. Cela a toutefois lieu non pas dans la moindre complémentarité mais dans un parfait semblant. C'est cette dimension de semblant, insigne dans la définition du phallus, que l'on s'empresse de laisser de côté lorsqu'on imaginarise la théorie.

Ne pourrait-on pas penser alors que c'est justement lorsqu'un des membres d'un couple tente d'échapper à ces prescriptions de genre du rapport au phallus, de défaire le semblant, que se produit la prise d'acte des situations de violence conjugale? Si la violence éclate avant que l'un des deux ne tente de sortir d'une dialectique phallique imaginarisée, la prise de conscience de cette violence apparaît lorsque le semblant se révèle, et que choient les identifications au «type idéal» plus que du sexe, du genre assigné au sujet.

Plutôt que d'accuser alors ces femmes d'être phalliques ou de n'avoir pas réglé son sort à leur rapport au phallus, ne convient-il pas de voir qu'elles viennent interroger une répartition genrée de ces rôles du semblant, et indiquer la fêlure de normes d'existence qu'elles ne partagent alors plus avec leur partenaire? 
Le cas d'une patiente suivie au Brésil, pourrait être soumis à une lecture par le phallus dont la fulgurance tiendrait à la rengaine. Il semble toutefois révéler qu'il y est question d'autre chose.

Voilà 33 ans que Sonia vit avec son partenaire, Walter, dont elle a 3 enfants, João, 30 ans, Simone, 24 ans, et Walter Jr, 11 ans. Walter est décrit comme un homme coléreux, peu apte à dialoguer, prompt à s'emporter et à la battre ou à battre ses enfants. Elle en est dépendante économiquement : s'il ne lui interdit pas de travailler, il rend toutefois cela malaisé pour elle. Sonia passe le plus clair de ses séances à pleurer, elle semble consumée par une culpabilité démesurée, qui a envahi tous les plans de sa vie : elle est coupable de ne pas avoir bien éduqué ses deux premiers enfants, d'être tombée enceinte tardivement du troisième, de ne pas savoir le protéger de la violence du père et des deux premiers, de savoir que son mari la trompe et de ne pas arriver à se confronter à lui sur ce point, et de bien d'autres auto-accusations. Elle souffre, par ailleurs, depuis quelques années, d'une maladie, très imprécisément décrite, lui rendant malaisées les relations sexuelles avec son mari.

C'est à nouveau la culpabilité, imaginaire assignation par le regard de l'autre ici, qui semble la déterminer à réagir : elle se résout à ne plus accepter la situation quand elle est informée par la voisine que son mari la trompe. Celui-ci travaille comme gardien de nuit dans un club échangiste, et a été vu plusieurs fois accompagné de clientes de l'établissement. La situation semble tout d'un coup insupportable, et ce regard de la voisine lui désignant l'infidélité du conjoint rend inacceptable la violence à laquelle elle est soumise, la pousse à consulter et à vouloir y changer quelque chose.

Les ingrédients seraient ici réunis : Sonia a prétendu, pendant 33 ans, être le phallus pour son mari, en trouvant en lui à la fois la visée d'un désir et d'une demande - il est 
celui qui comblerait son manque, puisqu'elle n'a pas à travailler, et par sa présence/absence porterait le foyer entier. Voilà pourquoi sa frigidité, convertie dans cette imprécis mal qui l'empêche d'avoir des relations sexuelles avec lui, serait tolérée. Lui, au contraire, trouvant la satisfaction de sa demande en elle, ne cède pas sur son désir du phallus en allant vers d'autres femmes, vierges ou moins vierges, refusant toute impuissance. S'il la bat, c'est probablement parce qu'il ne sait combler sa demande d'un plus-de-jouir échappant à la logique phallique.

Se cantonner à ce psittacisme théorique littéralisant reviendrait ici à perdre de vue l'essentiel. Pour Sonia l'assomption de cette situation de violence conjugale, la souffrance extrême qu'elle éprouve à prendre acte de ce mode d'échange avec son mari n'apparaissent véritablement que lorsqu'elle fait choir la captation imaginaire de ce semblant, interroge les assignations de genre ici présentes, ne supporte pas ce qu'elle présente comme l'ultime violence: qu'on ait vu son mari la tromper. Si ce regard rendant la situation insupportable procède d'enjeux narcissiques pour Sonia, soulignons ici qu'il a pour effet de défaire certaines évidences de genre jusqu'alors non questionnées par elle, et d'en briser la performativité.

\section{Conclusion}

Quelles perspectives serait-il alors judicieux de développer dans la théorisation analytique, pour penser à nouveaux frais les situations de violence conjugale? 
Contentons-nous d'indiquer ici quelques pistes.

- La perspective des théories du genre et la déconstruction du semblant qu'elles effectuent permettent de considérer la violence conjugale comme violence de genre, assignation de genre, perpétuée parfois par la théorisation elle-même.

- La notion butlerienne de mélancolie de genre rendrait possible une lecture de la violence. Celle-ci se déclencherait lorsque cet autre, pris dans une fonction sociale, sexuée et sexuelle, différente de celle de la/du partenaire, donne à voir ce sur quoi porte le deuil impossible de la sexuation.

- La catégorie d'identification projective s'avère ici particulièrement utile : comme pour le racisme ou l'homophobie, c'est un inassimilable en soi qui est ici battu en l'autre.

- La question de la neutralité bienveillante se pose eu égard à ces situations, lorsqu'une clinique de l'auto-destructivité surgit. Comment l'analyste supporte cela pour l'autre, sans décider ou désirer à la place de l'autre ? S'ensuit ici la nécessité d’un remaniement de la dimension transféro-contretransférentielle, tant clinique que théorique.

La théorie psychanalytique et les concepts qu'elle articule ne sont pas éternitaires. L'écriture psychanalytique ne vient pas s'ajouter aux autres théories, car elle ne fonctionne pas à leur manière. Plus qu'un corpus fixé, il s'agit, en psychanalyse, de mouvements théoriques, d'opérations analytiques, qu'il semble préférable de nommer méthode ou interprétation analytique plutôt que théorie. En toute théorie analytique réside un noyau pulsionnel, passionnel, propre à l'infantile du théoricien, un axe 
narcissique, lié ici aux assignations de genre, et que la clinique des violences conjugales semble révéler davantage.

Est-ce véritablement en psychanalystes que parlent alors ces auteurs, qui, au nom de la psychanalyse, gratifient la communauté de préférences subjectives bien peu soumises à l'analyse du contre-transfert, et reproduisent les stéréotypes les plus poussifs dans un habillage de métapsychologie éternitaire?

Parler comme psychanalyste et parler au nom de la psychanalyse sont deux postures très différentes, probablement peu compatibles. La première, celle du psychanalyste, implique le cheminement d'une analyse personnelle, dont une visée est la renonciation à abuser d'une position de pouvoir. La seconde, prétendant, au nom de la théorie psychanalytique, statuer sur des questions posées par des sujets ré-élaborant leurs identifications de genre est une position de pouvoir, et de toute-puissance. Et qu'est la violence, sinon ce recours ultime du pouvoir quand il perd toute légitimité ?

\section{Bibliographie}

Butler, J. 2005. Troubles dans le genre. Le féminisme et la subversion de l'identité. Paris: La découverte.

Derrida, J. 2000. États d'âme de la psychanalyse. Adresse aux États Généraux de la Psychanalyse, Gallilée. 
Fonseca, M. C., Isabel, S. M., \& Vanessa, C. S. 2001. Bate-se uma mulher- questões sobre a violência e o feminino. Reverso. Revista de psicanalise , 48.

Foucault, M. 1976. Histoire de la sexualité. La volonté de Savoir, Paris, Gallimard.

Freud, S. 1894-1924. Névrose, psychose, perversion, Paris, P.U.F.

Freud, S. 1907-1931. La Vie sexuelle, Paris, P.U.F.

Lacan, J. 1958. « La Signification du phallus », in Ecrits. Paris, Seuil.

Laplanche, J. 1980. Problématiques II. Castration, symbolisation, Paris, P.U.F.

Laplanche, J. 2003. Sexual. La sexualité élargie au sens freudien, Paris, P.U.F.

Schneider, M. 2000. Généalogie du masculin, Paris, Aubier.

Schneider, M. 2004. Le paradigme féminin, Paris, Aubier. 


\section{Résumé}

Cet article a pour visée d'analyser certaines théorisations psychanalytiques de la violence conjugale, à la lumière d'une expérience clinique auprès de femmes souffrant de violences conjugales dans la banlieue de São Paulo. Cette recherche s'inscrit dans une étude mettant en correspondance la psyché et le champ social.

Traduisant une indissociabilité entre recherche, clinique et théorisation, la psychanalyse n'est point d'abord une théorie mais une méthode liée à un objet, l'inconscient, rendu positif par une procédure de recherche et une technique.

Comment penser alors une théorisation susceptible de rendre compte de la singularité social-historique de la violence conjugale contre les femmes? Littéralisant ou imaginarisant des textes freudiens ou lacaniens, bien des interprétations psychanalytiques font état d'une position masochiste féminine essentialisée, ou d'une lecture réduite du phallus.

La violence conjugale contre les femmes repose, à nouveaux frais, la question de l'historicité de la théorisation, de son inscription sociale, culturelle, politique, autant d'interrogations que les études du genre ne manquent pas de formuler à la psychanalyse.

La confrontation à la signification clinique et historique de la violence conjugale contre les femmes est alors une occasion d'éviter qu'une métapsychologie figée, an-historique, ne devienne paradoxale résistance à la psychanalyse.

\section{Mots-clés}

violence conjugale, psychanalyse, théories du genre, théorisation, historicité

\section{Domestic Violence, Gender Violence. Psychoanalysis Questioned by Gender}

\section{Summary}

This article aims to examine certain psychoanalytical theories of domestic violence, confronting them to a clinical experience with abused women in suburban Sao Paulo. This perspective relies on a correspondence between the psyche and the social context. Research, clinical experience and theory are narrowly linked in psychoanalysis, which, more than a mere theory, proves to be a method applied to an objet, the Unconscious, made positive only through the psychoanalytical research and technique.

This article purports to analyse how a psychoanalytical theory of domestic violence could account for the specific social-historic dimension of this phenomenon.

Some psychoanalytical interpretations literalize or imaginarize Freudien and Lacanien texts, through notions of essentialized feminine masochism or a reductive reading of the phallus.

Domestic violence against women questions in a new way the historicity of theory, its social, cultural and political definitions. These are issues along which Gender Studies 
address psychoanalysis.

Taking into account the clinical and historical signification of domestic violence may help avoiding that frozen an-historical metapsychology become a paradoxical resistance against psychoanalysis.

\section{Key-words}

Domestic Violence, Psychoanalysis, Gender Theories, Theorizing, Historicity 